\title{
ORIGINAL
}

\section{Clinical characteristics of thyroid abnormalities induced by sunitinib treatment in J apanese patients with renal cell carcinoma}

\author{
Shuichi Sato ${ }^{1)}$, Kazuhisa Muraishi ${ }^{1)}$, Junichi Tani ${ }^{1)}$, Yuko Sasaki ${ }^{1)}$, Ichiro Tokubuchi ${ }^{1)}$, Yuji Tajiri ${ }^{1)}$, \\ Kentaro Yamada ${ }^{1)}$, Shigetaka Suekane ${ }^{2)}$, Jiro Miyajima ${ }^{2}$, Kei Matsuoka ${ }^{2)}$ and Yuji Hiromatsu ${ }^{1)}$ \\ ${ }^{1)}$ Division of Endocrinology and Metabolism, Department of Medicine, Kurume University School of Medicine, Fukuoka 830-0011, \\ Japan \\ ${ }^{2)}$ Department of Urology, Kurume University School of Medicine, Fukuoka 830-0011, Japan
}

\begin{abstract}
Sunitinib is a multi-targeted tyrosine kinase inhibitor that is effective for advanced renal cell carcinoma. However, sunitinib often causes hypothyroidism. In this study, we report eight cases with thyroid dysfunction that occurred during sunitinib treatment for advanced renal cell carcinoma. In seven cases, mild hypothyroidism developed early in the first treatment cycle, and recovered spontaneously. Transient hyperthyroidism was observed during the second or third treatment cycles and was preceded by a rapid increase in thyroglobulin levels. ${ }^{99 \mathrm{~m}} \mathrm{Tc}$ scintigraphy in the hyperthyroid state showed decreased thyroidal uptake of ${ }^{99} \mathrm{TcO}_{4}$, suggesting destructive thyroiditis. Hypothyroidism subsequently developed, requiring levothyroxine replacement therapy. Ultrasonography showed a hypoechogenic pattern of the parenchyma and decreased intrathyroidal blood flow. The thyroid glands ultimately became atrophic, which may progress to permanent hypothyroidism. These findings suggest that sunitinib-induced hypothyroidism may occur frequently and may be a consequence of thyroiditis with transient thyrotoxicosis. The marked decrease in thyroid size due to reduced capillary blood flow induced by VEGF receptor inhibition may cause delayed and/or permanent hypothyroidism. Therefore, thyroid function should be monitored in all patients treated with sunitinib.
\end{abstract}

Key words: Hypothyroidism, Renal cell carcinoma, Sunitinib, Thyrotoxicosis

SUNITINIB is an oral, small-molecule, multi-targeted receptor tyrosine kinase inhibitor that was approved by the US Food and Drug Administration for the treatment of advanced renal cell carcinoma and imatinibresistant gastrointestinal stromal tumor in January 2006 and by the Japan Ministry of Health, Labour and Welfare in June 2008 [1]. Sunitinib is now widely used for these indications worldwide. Sunitinib exerts anti-tumor and anti-angiogenesis activity by inhibiting several receptor tyrosine kinases, including vascular endothelial growth factor receptor (VEGFR1-3) and platelet-derived growth factor receptors (PDGFR $\alpha$ and PDGFR $\beta$ ) [2]. Several recent studies have report-

Received Apr. 28, 2010; Accepted Jul. 27, 2010 as K10E-130

Released online in J-STAGE as advance publication Aug. 21, 2010

Correspondence to: Shuichi Sato, Division of Endocrinology and Metabolism, Department of Medicine, Kurume University School of Medicine, 67 Asahi-machi, Kurume 830-0011, Japan.

E-mail: dem_sato@med.kurume-u.ac.jp ed sunitinib-induced hypothyroidism and thyroid dysfunction [2-11], although the underlying mechanism is not clear. The incidence of sunitinib-induced hypothyroidism has been reported to be 53-85\% [3, 4] and $36-46 \%[5,6]$ in retrospective and prospective studies, respectively. These rates are higher than those provided by Pfizer Inc. (16\% in Japan, $2.9 \%$ in other countries; SUT658001C).

Here, we describe eight consecutive patients who were treated with sunitinib for renal cell carcinoma, and who developed thyroid dysfunction during the treatment. We discuss in detail the clinical courses of two patients who exhibited thyroid dysfunction during sunitinib therapy.

\section{Patients and Methods}

In 2008-2009, eight patients with renal cell carcinoma received sunitinib therapy at the Department of 

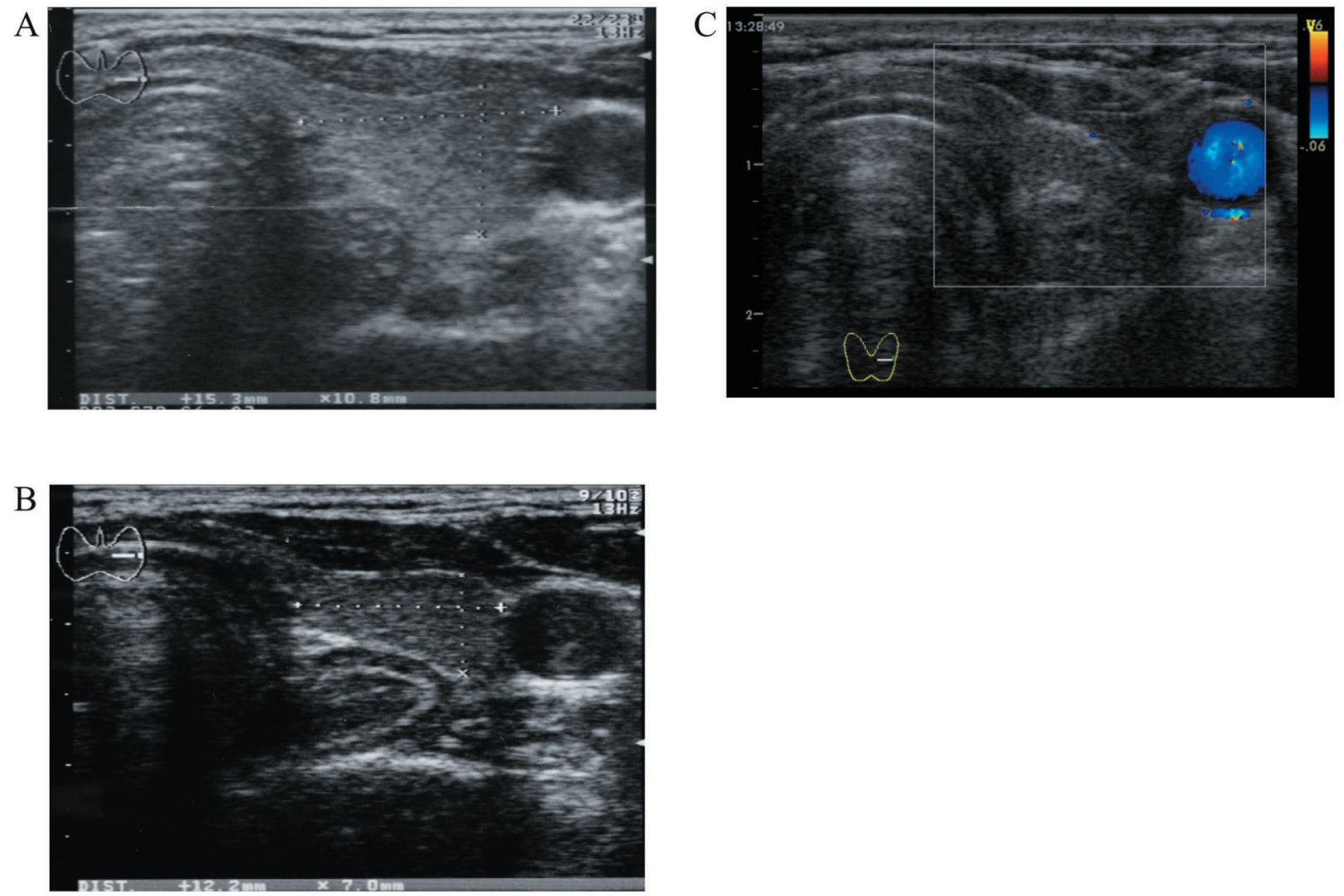

Fig. 1 Ultrasonography analysis of the thyroid gland in patient 1.

A: Before sunitinib therapy, thyroid gland appeared to be normal (size of the left lobe: $54.8 \times 15.3 \times 10.8 \mathrm{~mm}$; normal echogenicity). B: Six months later, thyroid gland was atrophic (size of the left lobe: $39.5 \times 12.2 \times 7.0$ mm; echogenicity, heterogenous). C: One year later, thyroid gland was atrophic and the intrathyroidal blood flow remarkably decreased.

Urology, Kurume University Hospital. The patients received repeated 4-week cycles of $50 \mathrm{mg}$ sunitinib per day, followed by a 2-week treatment withdrawal. Thyroid function tests were performed at on initiation of sunitinib therapy and were performed periodically thereafter. The protocol was approved by the Ethical Committee of the Kurume University School of Medicine and written consent was obtained by all the patients.

\section{Patient 1}

A 43-year-old Japanese female was diagnosed in May 2009 with renal cell carcinoma of the left kidney. She was referred to the thyroid clinic at Kurume University Hospital before starting sunitinib therapy to evaluate thyroid function. She was in a euthyroid state [free triiodothyronine (FT3) $2.9 \mathrm{ng} / \mathrm{dL}$, free thyroxine (FT4) $1.24 \mathrm{pg} / \mathrm{mL}$, TSH $1.25 \mathrm{mU} / \mathrm{L}$ ). Anti-thyroid antibodies were negative [anti-thyroglobulin antibody (TgAb) $1.5 \mathrm{U} / \mathrm{mL}$, anti-thyroid peroxidase antibody
(TPOAb) $<0.1 \mathrm{U} / \mathrm{mL}$, anti-human TSH receptor antibody (hTRAb) $<1.0 \mathrm{IU} / \mathrm{L}$, thyroid stimulating antibody (TSAb) 101\%]. Ultrasonography showed a normal thyroid gland (Fig. 1A).

Her serum TSH level gradually increased at 1 week after the first administration of sunitinib. Four weeks later, thyroid function tests showed mild hypothyroidism (FT3 1.7 ng/dL, FT4 0.74 pg/mL, TSH 27.68 $\mathrm{mU} / \mathrm{L})$. However, the TSH level recovered to a near normal level within the 2-week sunitinib withdrawal phase. During the second cycle of sunitinib therapy, her TSH level transiently increased to $24.5 \mathrm{mU} / \mathrm{L}$. Her serum Tg level increased from 8.6 to $480 \mathrm{ng} / \mathrm{dL}$ in week 3 . One week later, her thyroid hormone levels increased (FT3, from 3.4 to $5.1 \mathrm{ng} / \mathrm{dL}$; FT4, from 1.18 to $1.72 \mathrm{pg} / \mathrm{mL}$ ) and the TSH level decreased (from 7.03 to $0.128 \mathrm{mU} / \mathrm{L}$; Fig. 2). Ultrasonography showed a hypoechogenic pattern of the parenchyma and decreased intrathyroidal blood flow. These findings suggested that she developed destructive thyroiditis. 


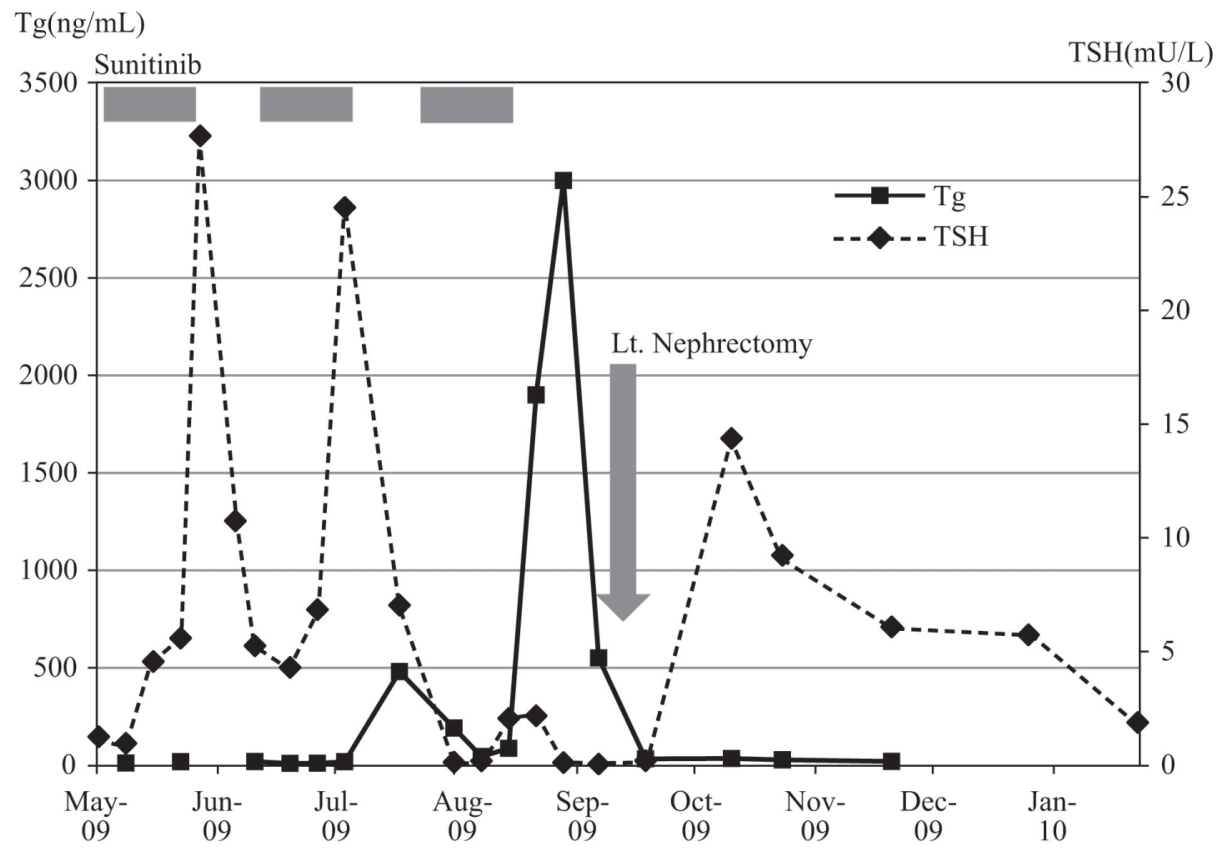

Fig. 2 Clinical course of patient 1

During the second withdrawal phase and at the start of the third treatment cycle, the Tg level decreased and the thyroid hormone and TSH levels were spontaneously normalized. Ultrasonography showed a normal pattern of the parenchyma and intrathyroidal blood flow. During the third cycle of sunitinib therapy, the Tg level rose markedly at week 3 (from 44 to $3000 \mathrm{ng}$ / $\mathrm{mL}$ ). One week later, FT3 and FT4 increased and the TSH level decreased. Ultrasonography showed decreased echogenicity in the parenchyma and reduced intrathyroidal blood flow.

Because the size of the renal cell carcinoma decreased after the third treatment cycle, she underwent left nephrectomy on September 28, 2009. She finally developed permanent hypothyroidism and therefore requires daily levothyroxine $(75 \mu \mathrm{g})$ therapy. Ultrasonography showed that the thyroid gland was atrophic with decreased echogenicity in the parenchyma and decreased intrathyroidal blood flow (Fig. 1B, 1C).

\section{Patient 2}

A 76-year old Japanese female was initially treated with sorafenib, another multi-targeted receptor tyrosine kinase inhibitor [12], for renal cell carcinoma with lymph node metastases, but it was discontinued after 5 weeks because of liver dysfunction with nausea and fatigue (AST $706 \mathrm{U} / \mathrm{L}$, ALT $1025 \mathrm{U} / \mathrm{L}$, ALP

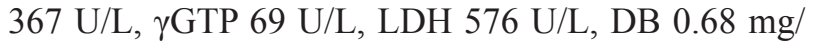
$\mathrm{dL})$. Thyroid function tests performed before starting sunitinib therapy showed subclinical hypothyroidism with increased TSH and normal FT4 levels. Her serum $\mathrm{TgAb}$ titer was $36.5 \mathrm{IU} / \mathrm{mL}$, suggesting the presence of chronic thyroiditis. She developed mild hypothyroidism during the first cycle of sunitinib therapy with increased TSH and decreased FT4 levels. Thus, she was treated with levothyroxine. She also developed thrombocytopenia 3 weeks after starting sunitinib, which recovered spontaneously during the 2-week withdrawal phase. In the middle of the third cycle, her serum Tg level rapidly increased (from 360 to $1800 \mathrm{ng} / \mathrm{mL}$ ) and she developed mild hyperthyroidism (Fig. 3). During the fourth cycle, her $\mathrm{Tg}$ level increased again (from 200 to $2100 \mathrm{ng} / \mathrm{mL}$ ) and she remained hyperthyroid. Therefore, levothyroxine therapy was discontinued. Her serum hTRAb and TSAb were negative throughout sunitinib therapy. During the fifth cycle, her serum $\mathrm{TgAb}$ titers increased to $207.3 \mathrm{IU} / \mathrm{mL}$ and she developed hypothyroidism, which required levothyroxine therapy $(62.5 \mu \mathrm{g} /$ day $)$. Her thyroid gland markedly decreased in size and her serum $\mathrm{Tg}$ levels were around $100 \mathrm{ng} / \mathrm{dL}$.

Subclinical hypothyroidism before sunitinib therapy was observed in three patients, who had been previously treated with sorafenib (Table 1). Five patients 


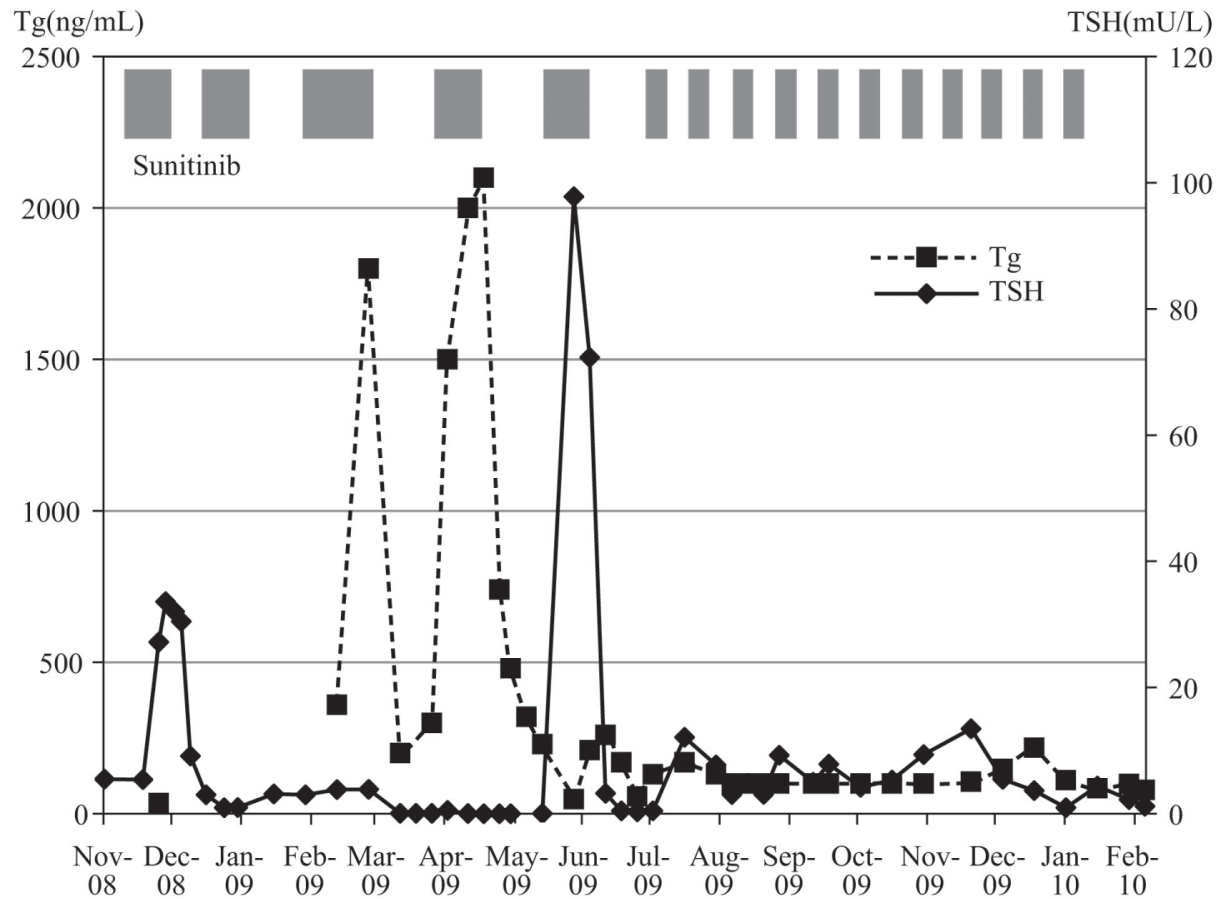

Fig. 3 Clinical course of patient 2

developed subclinical hypothyroidism and two patients developed clinical hypothyroidism during the first cycle of sunitinib therapy. However one patient (Table 1 Patient 8) developed thyrotoxicosis without the preceding hypothyroidism during the first sunitinib administration (Fig. 4).

Hyperthyroidism during sunitinib therapy was identified in seven patients based on low TSH and high FT4 and/or FT3 between the second and fourth cycles of sunitinib therapy. Furthermore, a rapid increase in Tg levels was observed in these patients. ${ }^{99 \mathrm{~m}}$ Tc scintigraphy showed decreased thyroidal uptake. The rapid increase in Tg level preceded the increases in FT3 and FT4 levels. Two months later, the magnitude of changes in Tg decreased and seven patients developed hypothyroidism, requiring levothyroxine therapy (50-100 $\mu \mathrm{g} /$ day at the end of the observation period). Ultrasonography showed that the thyroid glands were atrophic in seven patients. Only one patient (Table 1 Patient 7) developed subclinical hypothyroidism early in the first treatment cycle and remained subclinical hypothyroidism until she underwent nephrectomy after 6 cycles of sunitinib therapy.

The TgAb and TPOAb titers did not change throughout the treatment courses, except in one case (patient 2) who had TgAb at baseline (Table 1).
Color Doppler analysis showed the decrease of blood flow (vascularity) during on-sunitinib period (Fig. 4B) and restored withdrawal period (Fig. 4C). However, it markedly decreased at the permanent hypothyroid phase (Fig. 1C).

\section{Discussion}

Thyroid dysfunction was observed in all eight patients with advanced renal cell carcinoma treated with sunitinib described here. Thyroid function abnormalities, including hypothyroidism, occurred early in the treatment course and were followed by transient thyrotoxicosis and ultimately hypothyroidism, which necessitated replacement therapy. We also found significant changes in Tg levels during sunitinib therapy.

The incidence of sunitinib-induced hypothyroidism has been reported to be $53-85 \%$ in retrospective studies and $36-46 \%$ in prospective studies [3-6]. Although the number of patients reported here was small, the incidence of thyroid function abnormalities was quite high relative to these earlier reports.

Interestingly, we showed 3 phases of thyroid dysfunction (hypothyroidism at the early cycles of sunitinib treatment, and then transient thyrotoxicosis with subsequent delayed hypothyroidism and permanent 


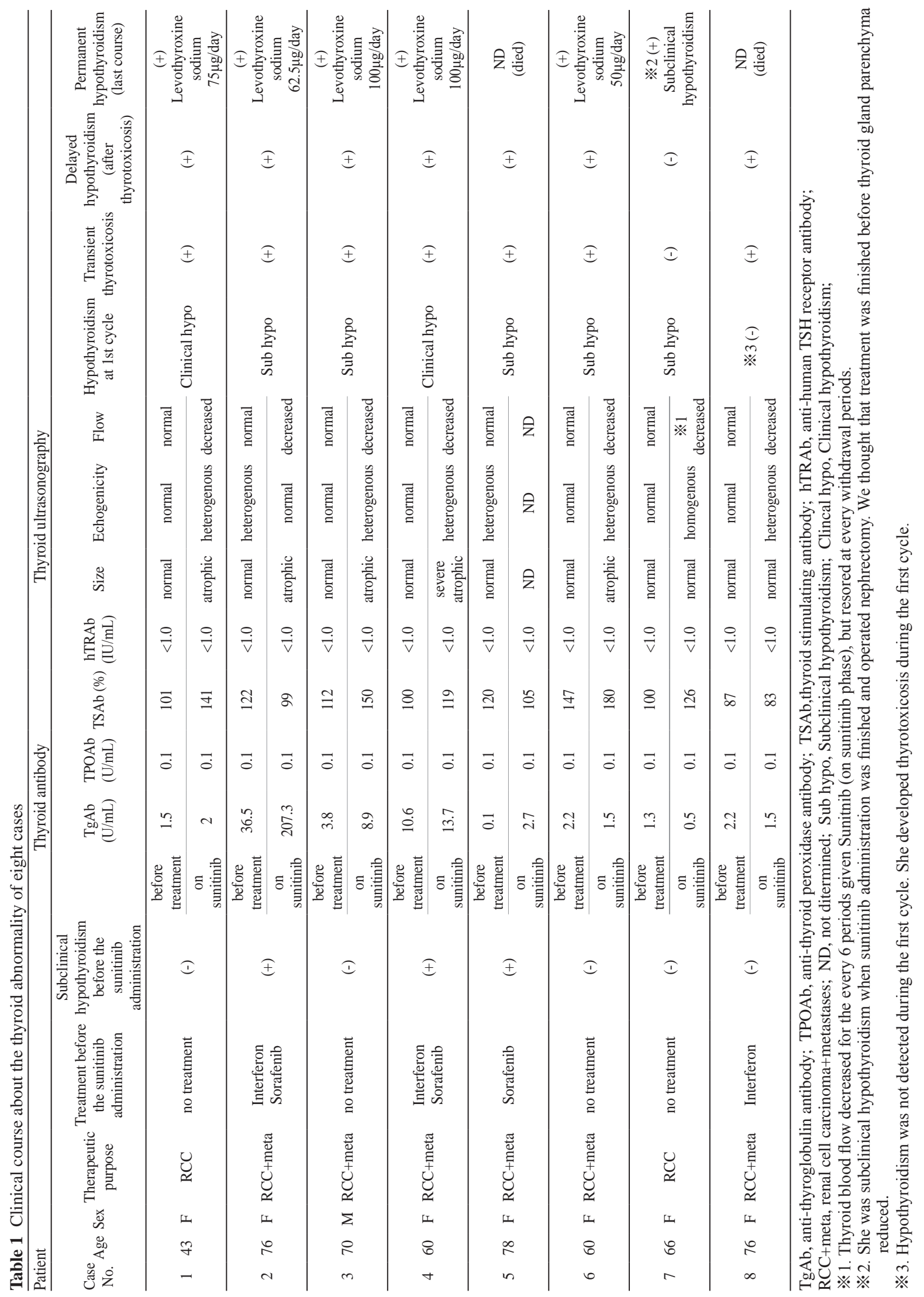



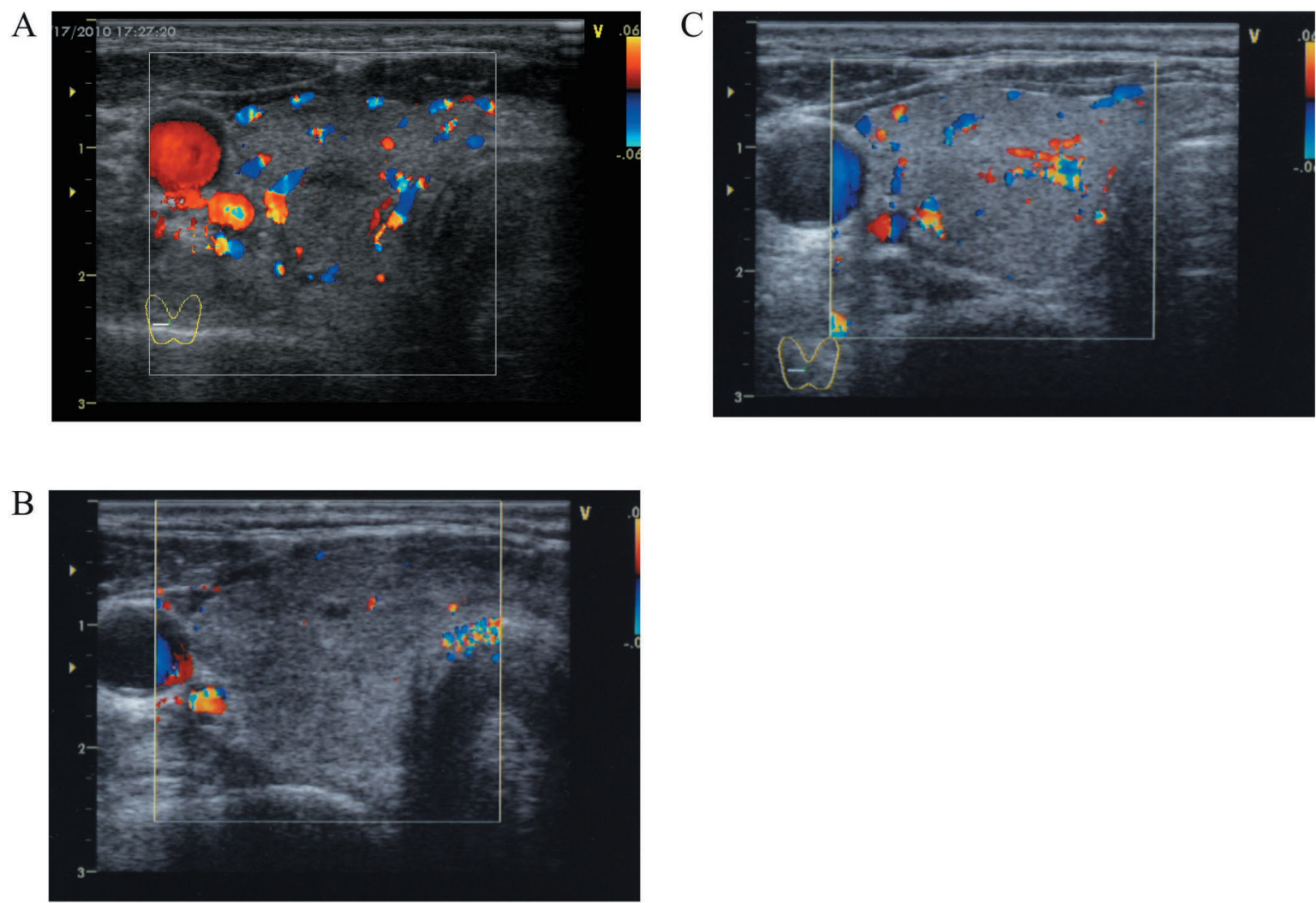

Fig. 4 Ultrasonography analysis of the thyroid gland in patient 8.

A: Before sunitinib therapy, the thyroid gland and intrathyroidal blood flow appeared to be normal. B: During the first cycle of the sunitinib administration with thyrotoxicosis, the thyroidal parenchyma became hypoechoic and its blood flow was remarkably decreased. C: During the sunitinib withdrawal phase (just after the first cycle), the intrathyroidal blood flow restored clearly.

hypothyroidism) in 5 of 8 patients. The differences in the interval of the thyroid function tests, and racial or individual susceptibility to sunitinib may explain the frequent occurrence of sunitinib-induced thyroid dysfunction in our series.

Various mechanisms have been hypothesized to explain tyrosine kinase inhibitor-induced hypothyroidism, including reduced synthesis of thyroid hormones due to inhibition of thyroid peroxidase activity [4], progressive depletion of functional reserves, inhibition of thyroidal uptake of iodine [6] through direct inhibition of fluorine contained in sunitinib on the sodium iodide symporter (NIS) [6] or the TSH receptor, or by drug-induced atrophy of the thyroid caused by inhibition of gland vascularity [3]. Angiogenesis is inhibited by inhibiting the tyrosine kinase activity of VEGFR1-3, PDGFR $\alpha$ and PDGFR $\beta$ [2]. Other important targets for sunitinib belong to the tyrosine kinase family and include stem cell growth factor (c-
KIT), fetal liver tyrosine kinase receptor 3, colonystimulating factor 1 (CSF-1), and ret proto-oncogene (RET).

Desai et al. [5] suggested that sunitinib may cause destructive thyroiditis because 6 out of 15 (40\%) hypothyroid patients in their study had low TSH levels before developing hypothyroidism. Grossmann et al. [11] reported transient thyrotoxicosis and subsequent hypothyroidism in 6 out of 25 patients with sunitinib treatments. We postulate that sunitinib-induced thyrotoxicosis occurs frequently in contrast to the previous reports $[5,11]$. It may be due to the frequent monitoring of thyroid function.

Furthermore, Desai et al. [5] reported that two hypothyroid patients had sonographically undetectable thyroid tissue. No cytological evidence of inflammatory tissue changes was provided, and the authors proposed that the hypothyroidism was due to sunitinib-induced follicular cell apoptosis. By contrast, 
Alexandrescu et al. [9] reported lymphocytic thyroiditis during sunitinib therapy, suggesting that sunitinib may trigger autoimmune processes in the thyroid gland. In our study, the anti-Tg and anti-TPO antibody titers did not change throughout the treatment course, except in one patient who had TgAb at baseline. The prevalence of TgAb or TPOAb in patients with thyroid dysfunction induced by sunitinib was low [3, 5-7]. There was no correlation between the presence of antibodies and the incidence and severity of thyroid function abnormalities [3]. Therefore, autoimmune abnormalities do not appear to contribute to thyroid function abnormalities caused by sunitinib.

Grossmann et al. [10] and Faris et al. [11] also reported the increase of $\mathrm{Tg}$ in thyrotoxicosis. The rapid rise in Tg preceded the changes in FT3 and FT4 levels in seven patients with thyrotoxicosis caused by sunitinib in our series. However, the magnitude of changes in Tg levels gradually decreased during subsequent cycles. During subsequent episodes of thyrotoxicosis, the Tg content in the thyroid gland may be small at that time. Those changes were consistent in seven patients. Because the serum Tg level appears to be a good mark- er for thyroid destruction and the thyroid reservoir, the significance of the increase in Tg during sunitinib therapy should be investigated in future studies.

Finally, the thyroid glands became atrophic and may result in delayed and/or permanent hypothyroidism. However, the mechanisms involved in sunitinib-induced hypothyroidism are still unclear. The reduction in capillary blood flow caused by VEGFR inhibition may explain the marked decrease in thyroid size [13-15]. Another mechanism involved in thyroid dysfunction may be the inhibition of thyroid peroxidase leading to reduced synthesis of thyroid hormone [4]. Further studies are needed to clarify the molecular mechanisms involved in sunitinib-induced thyroid dysfunction.

In conclusion, sunitinib-induced thyroid dysfunction may occur frequently. It may be composed of hypothyroidism at the early cycles of sunitinib treatment, and then transient thyrotoxicosis with subsequent delayed hypothyroidism and permanent hypothyroidism. Thyroid function, therefore, should be regularly monitored in all patients treated with sunitinib.

\section{References}

1. Torino F, Corsello SM, Longo R, Barnabei A, Gasparini G. (2009) Hypothyroidism related to tyrosine kinase inhibitors: an emerging toxic effect of targeted therapy. Nat Rev Clin Oncol 6: 219-228.

2. Ivy SP, Wick JY, Kaufman BM. (2009) An overview of small-molecule inhibitors of VEGFR signaling. Nat Rev Clin Oncol 6: 569-579.

3. Rini BI, Tamaskar I, Shaheen P, Salas R, Garcia J, Wood L, Reddy S, Dreicer R, Bukowski RM. (2007) Hypothyroidism in patients with metastatic renal cell carcinoma treated with sunitinib. J Natl Cancer Inst 99: 81-83.

4. Wong E, Rosen LS, Mulay M, Vanvugt A, Dinolfo M, Tomoda C, Sugawara M, Hershman JM. (2007) Sunitinib induces hypothyroidism in advanced cancer patients and may inhibit thyroid peroxidase activity. Thyroid 17: 351-355.

5. Desai J, Yassa L, Marqusee E, George S, Frates MC, Chen MH, Morgan JA, Dychter SS, Larsen PR, Demetri GD, Alexander EK. (2006) Hypothyroidism after sunitinib treatment for patients with gastrointestinal stromal tumors. Ann Intern Med 145: 660-664.

6. Mannavola D, Coco P, Vannucchi G, Bertuelli R, Carletto M, Casali PG, Beck-Peccoz P, Fugazzola L.
(2007) A novel tyrosine-kinase selective inhibitor, sunitinib, induces transient hypothyroidism by blocking iodine uptake. J Clin Endocrinol Metab 92: 3531-3534.

7. Wolter P, Stefan C, Decallonne B, Dumez H, Bex M, Carmeliet P, Schöffski P. (2008) The clinical implications of sunitinib-induced hypothyroidism: a prospective evaluation. Br J Cancer 99: 448-454.

8. Vetter ML, Kaul S, Iqbal N. (2008) Tyrosine kinase inhibitors and the thyroid as both an unintended and an intended target. Endocr Pract 14: 618-624.

9. Alexandrescu DT, Popoveniuc G, Farzanmehr H, Dasanu CA, Dawson N, Wartofsky L. (2008) Sunitinibassociated lymphocytic thyroiditis without circulating antithyroid antibodies. Thyroid 18: 809-812.

10. Grossmann M, Premaratne E, Desai J, Davis ID. (2008) Thyrotoxicosis during sunitinib treatment for renal cell carcinoma. Clin Endocrinol (Oxf) 69: 669-672.

11. Faris JE, Moore AF, Daniels GH. (2007) Sunitinib (sutent)-induced thyrotoxicosis due to destructive thyroiditis: a case report. Thyroid 17: 1147-1149.

12. Tamaskar I, Bukowski R, Elson P, Ioachimescu AG, Wood L, Dreicer R, Mekhail T, Garcia J, Rini BI. (2008) Thyroid function test abnormalities in patients with metastatic renal cell carcinoma treated with sorafenib. 
Ann Oncol 19: 265-268.

13. Baffert F, Le T, Sennino B, Thurston G, Kuo CJ, HuLowe D, McDonald DM. (2006) Cellular changes in normal blood capillaries undergoing regression after inhibition of VEGF signaling. Am J Physiol Heart Circ Physiol 290: H547-559.

14. Makita N, Miyakawa M, Fujita T, Iiri T.(2010)
Sunitinib induces hypothyroidism with a markedly reduced vascularity. Thyroid 20: 323-326.

15. Rogiers A, Wolter P, Op de Beeck K, Thijs M, Decallonne B, Schöffski P. (2010) Shrinkage of thyroid volume in sunitinib-treated patients with renal-cell carcinoma: a potential marker of irreversible thyroid dysfunction? Thyroid 20: 317-322. 